\title{
Protección, función hepática y respuesta inmune humoral de ovinos vacunados con mimotopos de catepsina L1 y L2 infectados experimentalmente con Fasciola hepatica
}

\author{
Protection, liver function and humoral immune response in sheep vaccinated with mimotopes of \\ cathepsin L1 and L2 experimentally infected with Fasciola hepatica
}

\author{
H Quiroz-Romero ${ }^{\mathrm{a}^{*}}$, D Correa ${ }^{\mathrm{b}}$, D Castillo-Mata ${ }^{\mathrm{c}}$, S Ortega-Vargas ${ }^{\mathrm{a}}$, A Villa-Mancera ${ }^{\mathrm{d}}$ \\ aDepartamento de Parasitología, Facultad de Medicina Veterinaria y Zootecnia, \\ Universidad Nacional Autónoma de México, México, D.F.

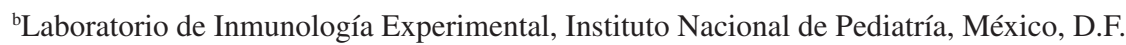 \\ 'Departamento de Patología, Facultad de Medicina Veterinaria y Zootecnia, \\ Universidad Nacional Autónoma de México, México, D.F. \\ dDivisión de Biotecnología Veterinaria y Biología Molecular, Facultad de Medicina Veterinaria y Zootecnia, \\ Benemérita Universidad Autónoma de Puebla, Tecamachalco Puebla, México.
}

\begin{abstract}
SUMMARY
Protection, liver function and humoral immune response were assessed in sheep vaccinated with mimotopes of cathepsin L1 and/or L2 and challenged with Fasciola hepatica metacercariae. Twenty sheep were randomly allocated in four groups of five animals each, for immunization with $1 \times 10^{14}$ phage particles of cathepsin L1 (YVYRWVEAECVA), cathepsin L2 (YVYEGRSRVRRP), cathepsin L1/L2 (YVYRWVEAECVA and YVYEGRSRVRRP) and phosphate buffered saline (PBS, control group), without adjuvant at weeks 0 and 2. All groups were challenged with 300 metacercariae at week 6 and slaughtered at week 22. Sheep vaccinated with cathepsin L1 mimotopes had a reduction in fluke burden of 50.11\% when compared to control group, whereas animals vaccinated with cathepsin L2 and L1/L2 the reduction was $35.82 \%$ and $13.85 \%$ respectively. This difference was not significant. Animals receiving cathepsin L2 and L1/L2 showed a significant reduction in eggs output. The sheep vaccinated with phage clones produced significantly higher titres of IgG antibodies than the sheep in the control group. No significant differences in serum glucose, levels of GLDH and GGT were observed between the control group and the vaccinated sheep.
\end{abstract}

Key words: Fasciola hepatica, sheep, phage display vaccine, cathepsin L.

\section{RESUMEN}

Protección, función hepática y respuesta immune humoral fueron evaluadas en ovinos vacunados con mimotopos de catepsina L1 y/o L2 y desafiados con metacercarias de Fasciola hepatica. Veinte ovinos fueron asignados al azar en cuatro grupos de cinco animales cada uno, para ser inmunizados con $1 \times 10^{14}$ partículas de fagos de catepsina L1 (YVYRWVEAECVA), catepsina L2 (YVYEGRSRVRRP), catepsina L1/L2 (YVYRWVEAECVA y YVYEGRSRVRRP) y amortiguador de fosfato salino (PBS, grupo control), sin adyuvante en las semanas 0 y 2 . Todos los grupos fueron desafiados con 300 metacercarias en la semana 6 y sacrificados en la semana 22. La vacunación de ovinos con mimotopos de catepsina L1, indujo una reducción de la carga parasitaria de 50,11\% en comparación con el grupo control, mientras que los animales vacunados con catepsina L2 y L1/L2 fue de 35,82\% y $13,85 \%$ respectivamente; esta diferencia no fue significativa. Los animales que recibieron catepsina L2 y L1/L2 mostraron una reducción significativa en la producción de huevos. Los ovinos vacunados con clonas de fagos producen títulos de anticuerpos IgG significativamente más altos que el grupo control. No hubo diferencias significativas en glucosa sérica, niveles de GLDH y GGT entre el grupo control y ovinos vacunados.

Palabras clave: Fasciola hepatica, ovinos, vacunación con fagos filamentosos, catepsina L.

\section{INTRODUCCIÓN}

La enfermedad parasitaria conocida como fasciolosis o distomatosis hepática es causada por el trematodo Fasciola hepatica en varias especies de mamíferos. En humanos, más de 90 millones de personas están en riesgo

Aceptado: 26.09.2013.

* hquiroz@unam.mx de infección y entre 2,4-17 millones de individuos están infectados con F. hepatica a nivel mundial (Keiser y Utzinger 2009). Esta enfermedad ha emergido como un importante patógeno en seres humanos en países como Bolivia, Perú, Ecuador, Egipto e Irán (O'Neill y col 1998, Rokni y col 2002, Mas-Coma y col 2005). Los hospederos más comunes de este helminto son los ovinos y los bovinos siendo una de las parasitosis más importantes en regiones templadas y tropicales en todo el mundo (Behn y Sangster 1999). La fasciolosis es una enfermedad para- 
sitaria de gran impacto económico, estimándose pérdidas por más de 3 mil millones de dólares por año (Spithill y col 1999).

El triclabendazol es la droga más efectiva contra estadios juveniles y maduros del parásito, se ha reportado ampliamente resistencia del helminto a este fasciolicida (Boray y col 1983, Fairweather 2009, Hanna y col 2013, Ortiz y col 2013). Otras medidas de control, particularmente vacunación, son necesarias para reducir la prevalencia de $F$. hepatica en animales domésticos, así como el riesgo zoonótico.

Fasciola hepatica secreta varias císteinas o tiol proteasas durante su ciclo de vida en los hospederos definitivos (Dalton y Heffernan 1989). Las catepsinas son las principales enzimas proteolíticas secretadas por el estadio larvario infectivo para atravesar la pared intestinal del hospedero (catepsinas B y L3), estadios migratorios para invadir tejido del hígado (catepsinas L1, L2, L3, L5 y B), así como parásitos adultos maduros que residen en los conductos biliares (catepsinas L1, L2 y L5) para alimentarse de sangre del hospedero (Kasný y col 2009, Robinson y col 2009, Morphew y col 2011, Stack y col 2011). También se ha demostrado que las proteasas del parásito participan en la producción de huevos y evasión de la respuesta inmune (Dalton y col 2003, Smooker y col 2010, McVeigh y col 2012).

Experimentos de vacunación en animales de laboratorio y rumiantes, utilizando moléculas purificadas nativas o recombinantes de catepsina $\mathrm{L}$ han demostrado inducir respuestas protectoras (McManus y Dalton 2006, Kesik y col 2007, Kašn y col 2009, Pérez-Ecija y col 2009, Golden y col 2010).

La tecnología de despliege en fagos o phage display es una herramienta útil para identificar péptidos que mimetizan epitopos de antígenos (mimotopos) (Devlin y col 1990, Sidhu y Koide 2007). Las bibliotecas de péptidos aleatorios han sido usadas exitosamante en el desarrollo de vacunas contra Plasmodium vivax (Demangel y col 1996), Plasmodium yoelii (Narum y col 2006), Schistosoma japonicum (Wu y col 2006), Taenia solium (Guo y col 2010, Gazarian y col 2012), Rhipicephalus microplus (Prudencio y col 2010) y Fasciola hepatica (VillaMancera y col 2008, Villa-Mancera y Méndez-Mendoza 2012). El objetivo del presente estudio fue evaluar el efecto de la vacunación de ovinos con mimotopos de catepsina L1 y L2 contra Fasciola hepatica sobre la carga parasitaria, producción de huevos, función hepática y respuesta inmune humoral.

\section{MATERIAL Y MÉTODOS}

\section{SELECCIÓN DE PÉPTIDOS DE UNA BIBLIOTECA DE DESPLIEGE EN FAGOS (PHAGE DISPLAY)}

Las clonas de fagos filamentosos fueron obtenidas como se ha descrito previamente (Villa-Mancera y col
2008). Los pozos de una placa de ELISA (Nunc-Immuno Plate F96, Cert, Maxisorp), fueron cubiertos con $150 \mu \mathrm{g} /$ $\mathrm{mL}$ de IgG anti-catepsina purificadas de conejo en $100 \mu \mathrm{l}$ de amortiguador de fosfato salino (PBS), $\mathrm{pH} 7,3$, y cubierta en un contenedor húmedo para evitar evaporación e incubada toda la noche en agitación a $4^{\circ} \mathrm{C}$. Posteriormente los pozos fueron bloqueados con $300 \mu \mathrm{l}$ de amortiguador de bloqueo (PBS conteniendo albumina sérica bovina [BSA] al $1 \%$ ) durante 1 hora a $4{ }^{\circ} \mathrm{C}$. Después de lavar seis veces con $200 \mu \mathrm{l}$ de PBS-T (PBS conteniendo Tween-20 al $1 \%$ ), se añadieron $1,0 \times 10^{11}$ partículas de fagos de una biblioteca combinatoria (M13) que expone aleatoriamente péptidos de 12 aminoácidos en la región $\mathrm{N}$-terminal fusionado con la proteína III (New England Biolabs, USA), en $100 \mu \mathrm{l}$ de PBS-T, incubándose por 1 hora a temperatura ambiente en agitación. Los fagos que no se unieron fueron removidos lavando con $200 \mu \mathrm{l}$ de PBS-T al $0,1 \%$ por 10 ocasiones. Los fagos que se unieron fueron incubados con $100 \mu \mathrm{l}$ de glicina- $\mathrm{HCl} 0,2 \mathrm{M}$, pH 2,2 por 10 minutos a temperatura ambiente y neutralizados con Tris- $\mathrm{HCl} 1 \mathrm{M}$, pH 9,0. Una pequeña fracción fue usada para titulación, el resto fue utilizado para infectar células de la cepa de $E$. coli ER2738 (New England Biolabs, USA) para la amplificación del fago. Se llevaron a cabo otras tres rondas de selección por afinidad, el primero utilizando $150 \mu \mathrm{g} / \mathrm{mL}$ de IgG purificada y por último utilizando 75,0 y $37,5 \mu \mathrm{g} /$ $\mathrm{mL}$ de IgG anti-catepsina purificada. Los fagos eluidos fueron adicionados a la cepa de E. coli ER2738 en el medio de cultivo $2 \mathrm{XYT}$, por 4,5 horas a $37^{\circ} \mathrm{C}$ en agitación constante y sembradas en una caja de Petri con agar LB y Xgal-IPTG. Las cajas Petri se incubaron toda la noche a $37^{\circ} \mathrm{C}$ y posteriormente 36 clonas fueron picadas al azar, cada clona seleccionada fue amplificada, purificada y titulada para ser secuenciada.

\section{PROTOCOLO DE VACUNACIÓN}

Veinte ovinos pelibuey de un año de edad fueron adquiridos del Centro de Enseñanza, Práctica e Investigación en Producción y Salud Animal de la Universidad Nacional Autónoma de México, y mostraron estar libres de la infección por análisis de heces (detección de huevos por la técnica de sedimentación) y anticuerpos contra los productos de excresión secreción (E/S) de F. hepatica en suero por ELISA (Villa-Mancera y col 2008). Los 20 ovinos fueron distribuidos al azar en 4 grupos de 5 animales. El grupo $1(\mathrm{n}=5)$ fue vacunado con $1 \times 10^{14}$ partículas de fagos de catepsina L1 (YVYRWVEAECVA), el grupo 2 $(\mathrm{n}=5)$ recibió $1 \times 10^{14}$ partículas de fagos de catepsina L2 (YVYEGRSRVRRP), el grupo $3(\mathrm{n}=5)$ fue inmunizado con $1 \times 10^{14}$ partículas de fagos de catepsina L1 y L2 (YVYRWVEAECVA y YVYEGRSRVRRP), y el grupo $4(\mathrm{n}=5)$ control recibió PBS estéril. Cada animal recibió la primera inyección por vía subcutánea y sin ningún adyuvante en la semana 0 . Una segunda inyección fue administrada a los 28 días (semana 4). 
TOMA DE MUESTRAS DE SANGRE

Se colectó sangre completa en tubos vacutainer y sin anticoagulante de la vena yugular de cada ovino, ésta fue centrifugada a $2.000 \mathrm{rpm} \mathrm{a} 4^{\circ} \mathrm{C}$ por 10 minutos y guardada a $-20^{\circ} \mathrm{C}$ hasta su uso. Las muestras fueron obtenidas cada 2 semanas desde la semana 0 y hasta el fin del experimento (semana 22).

\section{CONTEO DE HUEVOS EN HECES}

Las muestras de heces fueron colectadas semanalmente del recto de los animales inmunizados y del grupo control. Cinco gramos de cada muestra de heces fue procesada por la técnica de sedimentación como es descrita por Sexton y col (1990). Los huevos de $F$. hepatica fueron expresados como el número de huevos por gramo de heces (hpg).

\section{DESAFÍO CON PARÁSITOS}

Las cercarias de F. hepatica fueron liberadas de caracoles Lymnaea cubensis y guardadas a $4^{\circ} \mathrm{C}$. Las cápsulas de gelatina conteniendo las metacercarias (menos de 2 meses de edad) fueron administradas per os a cada animal con unas pinzas esofágicas. Cada ovino recibió 300 metacercarias 14 días después de la segunda inmunización (semana 6).

\section{MEDICIÓN DE LA PROTECCIÓN}

Todos los ovinos fueron sacrificados en la semana 22 posterior a la primera inmunización. En la necropsia, las vesículas biliares fueron abiertas en búsqueda de helmintos, los conductos biliares del hígado fueron cortados y los trematodos retirados con pinza roma. El hígado se cortó en trozos pequeños para la recolección de los parásitos restantes.

\section{ACTIVIDAD ENZIMÁTICA Y GLUCOSA SÉRICA}

Glutamato deshidrogenasa (GDH, indicativo de daño a parénquima hepático), gamma glutamil transferasa (GGT, indicativo de daño a conductos biliares) y glucosa fueron determinadas utilizando kits comerciales (Randox Laboratories Ltd, Antrim, UK; Genzyme Diagnostics, Cambridge, MA, USA), en un analizador de química Vitalab Selectra Junior (Vital Scientific, The Netherlands).

\section{RESPUESTA INMUNE HUMORAL INDUCIDA POR MIMOTOPOS ANTI-CATEPSINA L1 Y L2}

Los pozos de una placa de ELISA fueron cubiertos con $10^{10}$ partículas de fagos que fueron diluidos en PBS/BSA $0,2 \%$ e incubados toda la noche a $4{ }^{\circ} \mathrm{C}$ en agitación. Los pozos fueron lavados en cuatro ocasiones con PBS/Tween-20 al $0,1 \%$ y bloqueados con $200 \mu \mathrm{l}$ de PBS conteniendo BSA al $1 \%$ por 1 hora a $37^{\circ} \mathrm{C}$. Nuevamente fueron lavados los pozos en cuatro ocasiones. Posteriormente se incubó suero de ovino inmunizado a una dilución de 1:400 en PBS/BSA $0,2 \%$-Tween $0,2 \% 1$ hora a $37^{\circ} \mathrm{C}$. Se adicionó el anticuerpo anti-ovino-HRP (Serotec Ltd, Oxford, UK) en amortiguador de bloqueo (PBS/BSA 1\%) en un volumen final de $200 \mu \mathrm{l}$ por pozo, incubándose durante 1 hora a temperatura ambiente sin agitación. La unión específica de los fagos fue visualizada utilizando Tetrametilbenzidina (TMB, Sigma, USA), utilizando un volumen de $200 \mu \mathrm{l}$ por pozo. Se utilizó como control negativo PBS. La absorbancia fue determinada a $450 \mathrm{~nm}$ en un lector de ELISA (Biotek ELx800). Así mismo, para los productos de $\mathrm{E} / \mathrm{S}(30 \mu \mathrm{g} / \mathrm{mL})$ se empleó un ELISA indirecto (descrito anteriormente) con una dilución de suero de ovino de 1:800.

\section{ANÁLISIS ESTADÍSTICO}

Las medias aritméticas y desviación estándar fueron calculadas para la carga parasitaria, número de huevos en heces, actividad enzimática (GGT y GDH), glucosa y valores de los ELISA de los grupos vacunados y control. Se utilizó la prueba de Kolmogorov-Smirnov para confirmar normalidad. Para comparar los resultados entre grupos, se utilizó una prueba t de Student cuando la distribución fue normal y U de Mann-Whitney cuando la distribución no fue paramétrica. Para todas las pruebas se utilizó el programa IBM SPSS versión 20 para Windows (SPSS Inc., Chicago, USA). Una $\mathrm{P} \leq 0,05$ fue considerada como significativa.

\section{RESULTADOS}

\section{CARGA PARASITARIA DE ANIMALES INMUNIZADOS CON MIMOTOPOS DE CATEPSINA L}

Veintidós semanas después del inicio del experimento, los animales fueron sacrificados y los parásitos presentes en la vesícula biliar e hígado fueron contados (figura 1). El porcentaje de fasciolas recuperadas del grupo control fue en promedio $30,33 \%$ del total de 300 metacercareas administradas en la semana 6 del experimento. La inmunización de ovinos usando clonas de mimotopos de catepsina L1 sin adyuvante resulta en porcentajes de reducción significativas de la carga parasitaria de 50,11\% $(\mathrm{P}<0,05)$, comparado con el grupo control. Sin embargo, el grupo de animales inmunizados con catepsina L2 y una combinación de catepsina L1 y L2 no indujeron una protección significativa $(35,82 \%$ y $13,85 \%, \mathrm{P}>0,05)$.

\section{CONTEO DE HUEVOS EN HECES}

Se colectaron muestras de heces de ovinos inmunizados para su análisis coproparasitoscópico semanalmente, a partir de la semana 0 y hasta el sacrificio (semana 22). Los primeros huevos aparecen en las heces de los ovinos a partir de la semana 10 posterior a la infección con metacercarias del parásito (figura 2). El análisis de la producción 


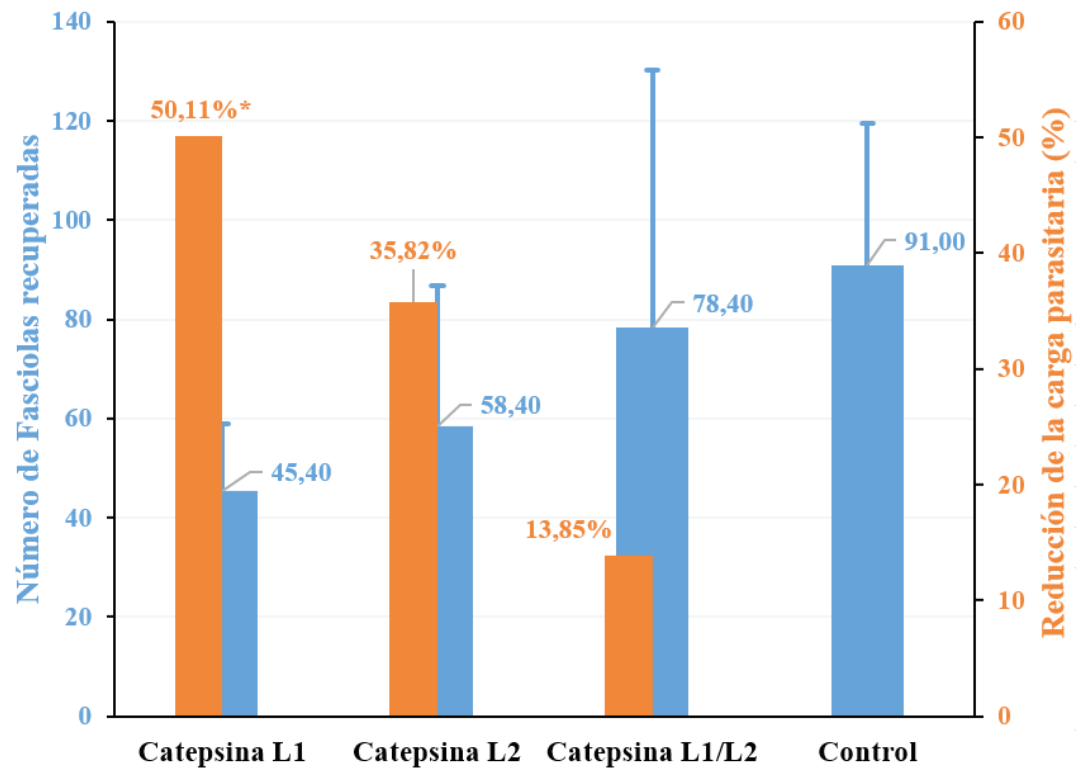

Figura 1. Reducción de la carga parasitaria de ovinos vacunados con mimmotopos de catepsina L de Fasciola hepatica. *Diferencias significativas en comparación con el grupo control $(\mathrm{P}<0,05)$. group $(\mathrm{P}<0.05)$.

Reduction of parasite burden of sheep vaccinated with cathepsin L mimotopes of Fasciola hepatica. * Significant differences versus control

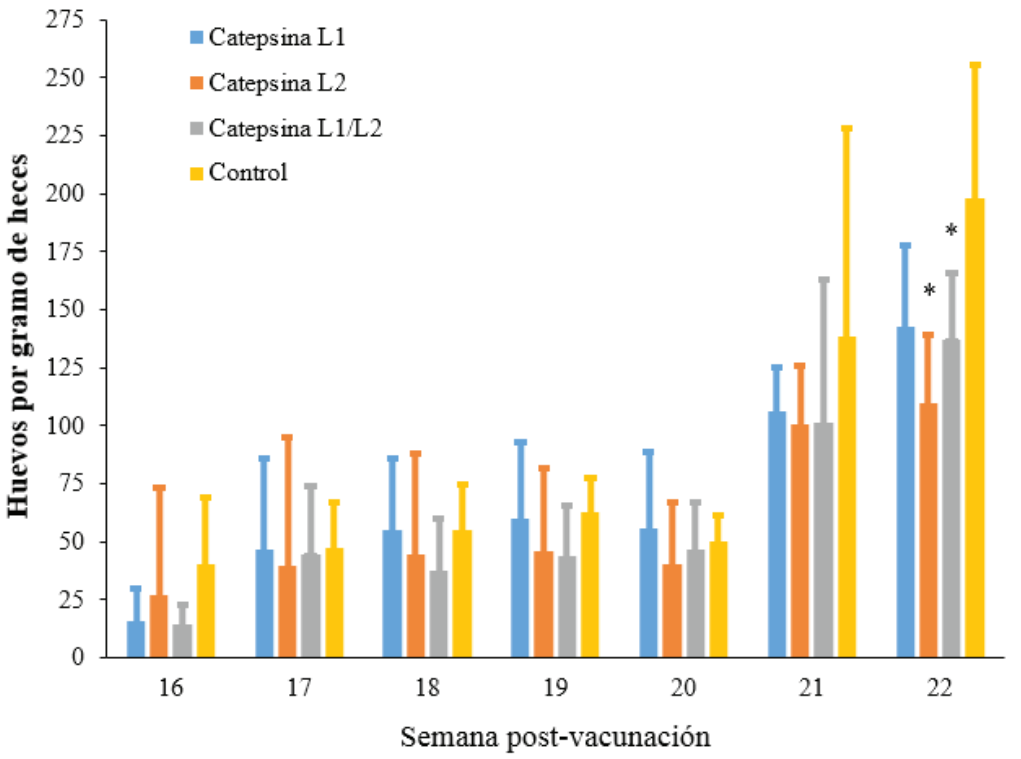

Figura 2. Media geométrica del número de huevos en heces de cada grupo durante el período de estudio. *Diferencias significativas en comparación con el grupo control $(\mathrm{P}<0,05)$.

Geometric mean fecal egg counts from each group during the study period. *Significant differences versus control group $(\mathrm{P}<0.05)$.

de huevos de $F$. hepatica por gramo de heces muestra una reducción significativa del $44,50 \%(\mathrm{P}<0,05)$ para el grupo vacunado con catepsina L2 en la semana 22 del estudio, comparado con el grupo control. Para el grupo de ovinos inmunizados con catepsina $L 1$, la reducción promedio fue del 27,85\%, y para catepsina L1/L2 30,78\% (P<0,05).

\section{RESPUESTA INMUNE HUMORAL INDUCIDA POR MIMOTOPOS DE CATEPSINA L}

La figura 3a muestra la dinámica de la respuesta de los anticuerpos contra los fagos filamentosos al utilizar en el ELISA como antígeno los productos de E/S de F. hepa- 

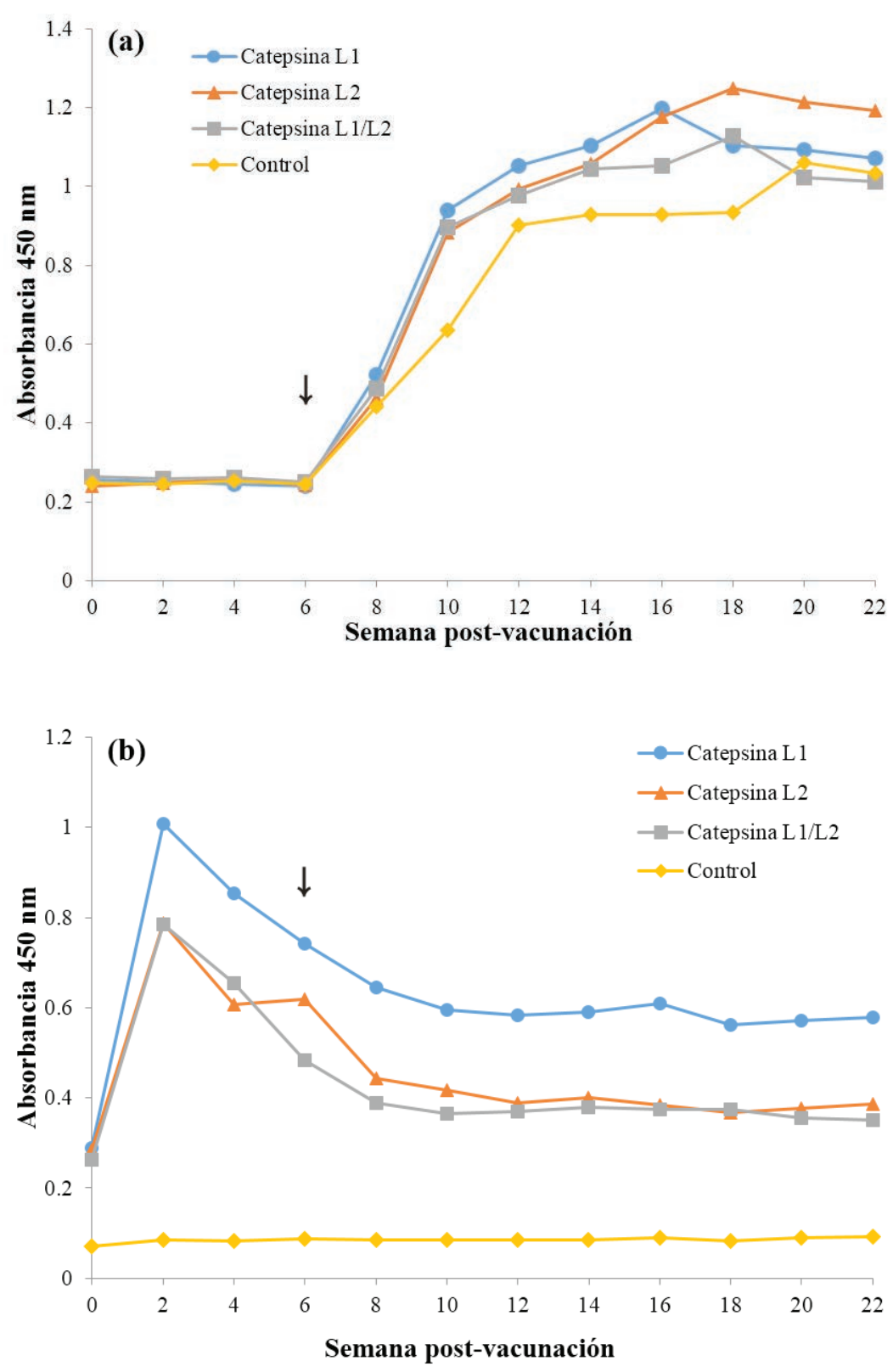

Figura 3. Análisis por ELISA de la IgG inducida por mimotopos de catepsina L1, L2 y L1/L2. Se utilizó como antígeno los productos de E/S de F. hepatica (a) y las clonas empleadas para la vacunación de ovinos (b). Los ovinos fueron inmunizados con 1 x $10^{14}$ partículas de fagos sin adyuvante, en la semana 0 y 4 . La flecha indica la semana de la infección experimental. Cada punto representa la media de 5 animales.

Analysis by ELISA of IgG induced by mimotopes of cathepsin L1 and/or L2. Excretion/secretion products was used as antigen (a), and clones used for vaccination of sheep. Sheep were given two immunizations at 0 and 4 week with 1 x $10^{14}$ phage particles without adjuvant. Week of experimental infection is indicated by the arrow.

tica. Una baja respuesta de anticuerpos aparecen en el suero de ovinos inmunizados dentro de las 6 semanas posteriores a la primera inmunización y muestran un marcado incremento después de la infección con metacercarias en la semana 6. Los niveles de respuesta de los anticuerpos inducidos por los mimotopos de catepsina L1 y/o L2 fueron más elevados que los producidos en el grupo control.
Los valores de las absorbancias de los ELISA muestran que los ovinos inmunizados con los mimotopos de catepsina L1, L2 y L1/L2, desarrollan anticuerpos IgG circulantes contra los fagos filamentosos, alcanzando un pico máximo dos semanas después de la primera inmunización (figura 3b). Esta respuesta desciende y permanece relativamente constante hasta el fin del experimento (semana 22). Se observó una fuerte respuesta de anticuerpos 
inducidos en animales vacunados con catepsina L1. Los ovinos vacunados con catepsina L2 y L1/L2 mostraron una menor respuesta inmune humoral.

\section{ACTIVIDAD ENZIMÁTICA Y GLUCOSA SÉRICA}

La actividad plasmática de las enzimas GLDH y GGT, así como glucosa permanecen dentro del rango normal para todos los grupos previo al desafio con metacercarias de $F$. hepatica en la semana 6 (figura $4 a-c$ ). La actividad de la GGT se eleva dos semanas después de la infección con los parásitos, alcanzando un pico en la semana 16 y disminuyendo hasta la semana 22. Dos semanas después del desafío (semana 6), existe un pequeño incremento en la actividad de la GLDH, alcanzando un nivel máximo en la semana 10, reduciéndose posteriormente estos valores hasta el final del experimento. Los niveles de glucosa permanecen relativamente constantes hasta la semana 10, disminuyendo estos valores entre la semana 12 y 14, aumentando al final del estudio. No hubo diferencias significativas entre los grupos de animales vacunados y el grupo control.

\section{DISCUSIÓN}

Existen numerosos estudios utilizando moléculas nativas purificadas o recombinantes como candidatos de vacunación contra F. hepatica (Hillyer 2005, Manus y Dalton 2006). Entre estas moléculas se encuentran las catepsinas L, las cuales son secretada en abundantes cantidades por el trematodo para facilitar la sobrevivencia en el hospedero, por lo cual es deseable aislar los motivos de inmunización con propósitos de vacunación. Mientras que las catepsinas $\mathrm{L}$ nativas pueden ser purificadas en cantidades suficientes para pruebas experimentales, una producción a gran escala requiere un sistema factible (Dalton y col 2003). Las bibliotecas de fagos filamentosos han sido empleadas para seleccionar péptidos que mimetizan mimotopos de antígenos y que pueden ser utilizados como candidatos en el desarrollo de vacunas en el área de parasitología (Ellis y col 2012).

La vacunación de ovinos con dos mimotopos de catepsina (L1 y L2) y un mimotopo de catepsina L2 ubicado en la región media y amino terminal de la molécula nativa no se ha reportado. La inmunización de ovinos con mimotopos de catepsina L1 y L2 indujeron niveles de protección de 50,11 y 35,82\% respectivamente, cuando éstas fueron administradas separadamente; el alineamiento con la catepsina L1 y L2 madura sugiere ser un epitopo conformacional, por lo que los anticuerpos contra este péptido pueden estar jugando directamente un papel protector. La unión de la IgG de conejo anti-catepsina L1 a la proteasa in vitro resulta en la inhibición de la capacidad para romper moléculas de IgG que median la unión de los eosinófilos al parásito (Smith y col 1994).

Además, se observó una reducción en el número de huevos en heces de 27,85 a 44,50\% en animales vacu- nados con los bacteriófagos. La actividad de catepsina $\mathrm{L}$ posiblemente fue bloqueada debido a la inducción de anticuerpos afectando la producción de huevos. La inmunización con cada mimotopo de catepsina L1 y/o L2 indujo un efecto único en la carga parasitaria y el número de huevos producidos. En el primer reporte de vacunación en ovinos con catepsina L nativa, el número de huevos en heces se redujo en $69,7 \%$, sin tener un efecto sobre la carga parasitaria (Wijffels y col 1994). La inmunización de ovinos con catepsina L1 o L2 nativa contra $F$. hepatica indujo niveles de protección de 33 y $34 \%$ en la carga parasitaria, así como reducciones en el número de huevos producidos de 71 y $81 \%$ respectivamente; mientras que la administración en conjunto de catepsina L1 y L2 indujo una reducción del $60 \%$ de la carga parasitaria (Piacenza y col 1999). La expresión de catepsina L1 recombinante en Pichia pastoris para pruebas de vacunación indujo protección de 35 a $45 \%$ en borregos con un efecto en la fecundidad del parásito de 50\% (McManus y Dalton 2006). El nivel de protección inducido con los mimotopos de fagos filamentosos estuvo dentro del rango reportado con el uso de otros inmunógenos más grandes y costosos.

Ovinos infectados experimentalmente con metacercarias de $F$. hepatica han mostrado porcentajes de implantación entre 26,4 y 39,0\% (Wijffels y col 1994, Piacenza y col 1999, Raadsma y col 2007); en este estudio, el porcentaje de implantación promedio en el grupo control fue de $30,33 \%$. Para los grupos de animales vacunados con catepsina L1, L2 y L1/L2 los porcentajes de implantación $(15,13,19,46$ y 26,13\% respectivamente) fueron similares a los reportados en trabajos previos (rango de 14,6 a 26,4\%) (Wijffels y col 1994, Piacenza y col 1999, Villa-Mancera y Méndez-Mendoza 2012).

Las clonas de catepsina L utilizadas en este estudio inducen una respuesta alta de IgG y de magnitud significativa contra los productos de E/S. Además, el desafío con metacercarias indujo un aumento en la respuesta en esos grupos, lo cual sugiere que los niveles de anticuerpos fueron amplificados en respuesta a linfocitos B de memoria y que estos epitopos existen en la molécula nativa. Por otro lado, los anticuerpos producidos en respuesta a las clonas, inducen una respuesta de $\operatorname{IgG}$ característica posterior a la primera inmunización, alcanzando los títulos un pico en la semana 2 y disminuyendo hasta el final del experimento. Sin embargo, sólo se observó un segundo pico característico, posterior a una segunda inmunización o refuerzo en el grupo de ovinos vacunados con catepsina L2.

Animales infectados con metacercarias de F. hepatica producen IgG1, elicitando una respuesta TH2 no protectora (Mulcahy y col 1999, O'Neill y col 1998, Hoyle y col 2003). La subclase de anticuerpos (IgG1, IgG2) producidos posterior a la vacunación, puede determinar el mecanismo efector para eliminar la infección. Ovinos y bovinos vacunados con catepsina $\mathrm{L}$ tienen una correlación positiva entre carga parasitaria y títulos altos de 

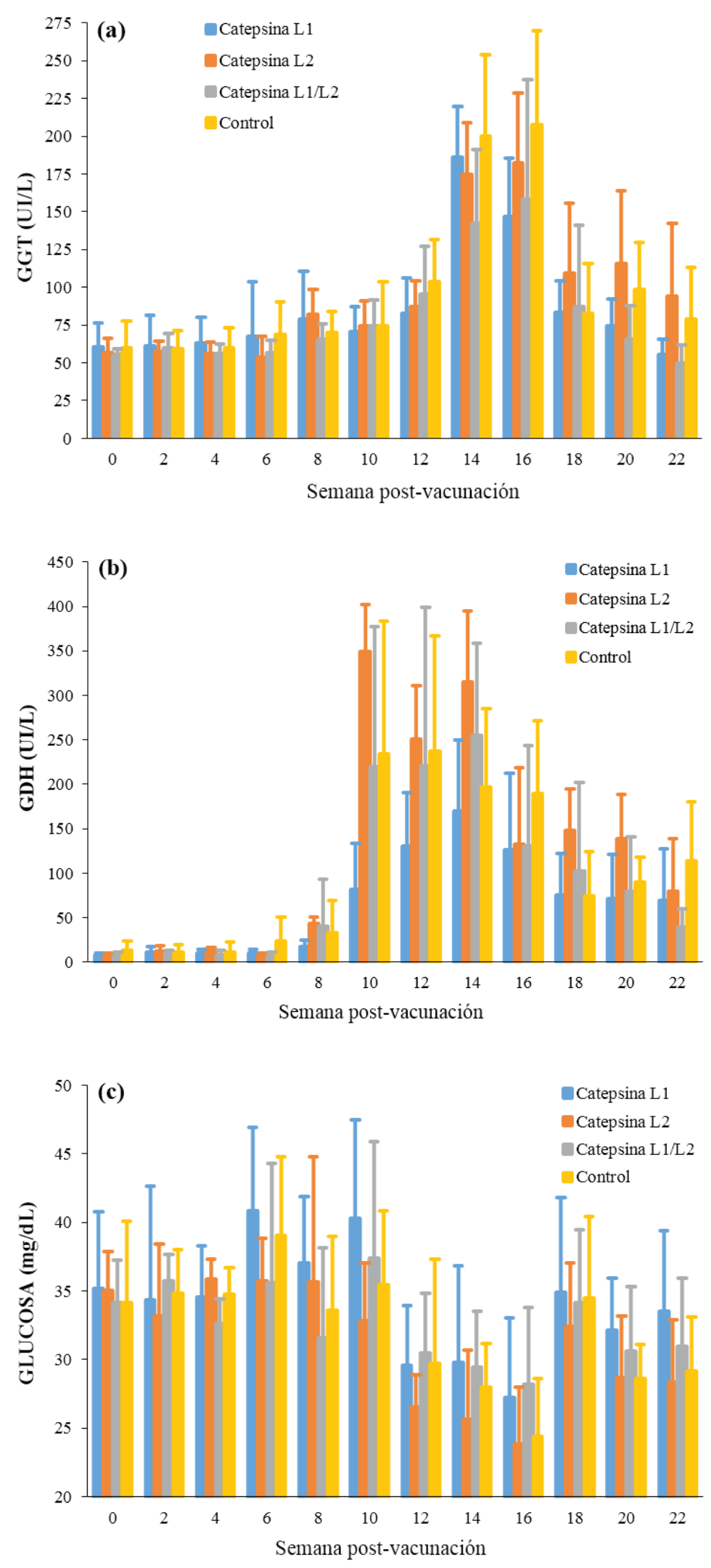

Figura 4. Análisis cinético de las actividades de gamma glutamil transferasa (GGT) (a), glutamato deshidrogenasa (GDH) (b) y glucosa (c) de animales vacunados con mimotopos de catepsina L y PBS (grupo control).

Kinetic analysis of activities of gamma glutamyl transferase (GGT) (a), glutamate dehydrogenase (GDH) (b) and glucose (c) of animals vaccinated with cathepsin L mimotopes and PBS (control group). 
anticuerpos $\operatorname{IgG} 1$ e $\operatorname{IgG} 2$, indicando que la protección está asociada con la inducción de una respuesta TH1 o balance Th1/Th2 (Mulcahy y col 1998, Villa-Mancera y Mendez-Mendoza 2012).

Los datos presentados demuestran que los mimotopos de catepsina $L$ inducen una respuesta immune protectora contra la infección de $F$. hepatica. Estos resultados son alentadores en términos de desarrollo de vacunas debido a la simplicidad relativa en la selección y corta longitud de los péptidos. La potenciación de la respuesta immune a los mimotopos utilizando adyuvantes apropiados podría ser investigada. Además, las clonas de fagos de la misma enzima o de diferentes moléculas como antígenos pueden ser usados en conjunto para incrementar el nivel de protección. Por otro lado, la reducción de la carga parasitaria, número de huevos en heces pueden reducir la morbilidad y mortalidad en el ganado. Una diseminación baja de huevos puede tener un efecto negativo en la contaminación de pasturas y en este sentido en la prevalencia de fasciolosis en zonas endémicas.

\section{AGRADECIMIENTOS}

Los autores agradecen a los Proyectos DGAPA-PAPIITUNAM IT230011 y BUAP-VIEP (VIMA-NAT-14-I).

\section{REFERENCIAS}

Behm CA, NC Sangster. 1999. Phathology, pathophysiology and clinical aspects. In: Dalton JP (ed). Fasciolosis. CABI Publishing, Oxon, UK, Pp 185-224.

Boray JC, PD Crowfoot, MB Strong, JR Allison, M Schellenbaum, M Von Orelli, G Sarasin. 1983. Treatment of immature and mature Fasciola hepatica infections in sheep with triclabendazole. Vet Rec 113, 315-317.

Dalton JP, M Heffernan. 1989. Thiol proteases released in vitro by Fasciola hepatica. Mol Biochem Parasitol 35, 161-166.

Dalton JP, SO Neill, C Stack, P Collins, A Walshe, M Sekiya, S Doyle, G Mulcahy, D Hoyle, E Khaznadji, N Moiré, G Brennan, A Mousley, N Kreshchenko, AG Maule, SM Donnelly. 2003. Fasciola hepatica cathepsin L-like proteases: biology, function, and potential in the development of first generation liver fluke vaccines. Int J Parasitol 33, 1173-1181.

Demangel C, P Lafaye, JC Mazie. 1996. Reproducing the immune response against the Plasmodium vivax merozoite surface protein 1 with mimotopes selected from a phagedisplayed peptide library. Mol Immunol 33, 909-916.

Devlin JJ, LC Panganiban, PE Devlin. 1990. Random peptide libraries: a source of specific protein binding molecules. Science 249, 404-406.

Ellis SE, GF Newlands, AJ Nisbet, JB Matthews. 2012. Phage display library biopanning as a novel approach to identifying nematode vaccine antigens. Parasite Immunol 34, 285-295.

Fairweather I. 2009. Triclabendazole progress report, 20052009: an advancement of learning?. J Helminthol 83, 139-150.
Gazarian K, M Rowlay, T Gazarian, JE Vazquez Buchelli, M Hernández Gonzáles. 2012. Mimotope peptides selected from phage display combinatorial library by serum antibodies of pigs experimentally infected with Taenia solium as leads to developing diagnostic antigens for human neurocysticercosis. Peptides 38, 381-388.

Golden O, RJ Flynn, C Read, M Sekiya, SM Donnelly, C Stack, JP Dalton, G Mulcahy. 2010. Protection of cattle against a natural infection of Fasciola hepatica byvaccination with recombinant cathepsin L1 (rFhCL1). Vaccine 28, 55515557.

Guo A, X Cai, W Jia, B Liu, S Zhang, P Wang, H Yan, X Luo. 2010. Mapping of Taenia solium TSOL18 antigenic epitopes by phage display library. Parasitol Res 106, 11511157.

Hanna RE, FI Forster, GP Brennan, I Fairweather. 2013. Fasciola hepatica: histological demonstration of apoptosis in the reproductive organs of flukes of triclabendazole-sensitive and triclabendazole-resistant isolates, and in fieldderived flukes from triclabendazole-treated hosts, using in situ hybridisation to visualise endonuclease-generated DNA strand breaks. Vet Parasitol 191, 240-251.

Hillyer GV. 2005. Fasciola antigens as vaccines against fascioliasis and schistosomiasis. J Helminthol 79, 241-247.

Hoyle DV, JP Dalton, M Chase-Topping, DW Taylor. 2003. Preexposure of cattle to drug-abbreviated Fasciola hepatica infections: the effect upon subsequent challenge infection and the early immune response. Vet Parasitol 111, 65-82.

Kasný M, L Mikes, V Hampl, J Dvorák, CR Caffrey, JP Dalton, P Horák. 2009. Peptidases of trematodes. Adv Parasit 69, 205-297.

Keiser J, J Utzinger. 2009. Food-borne trematodiases. Clin Microbiol Rev 22, 466-483.

Kesik M, L Jedlina-Panasiuk, M Kozak-Cieszczyk, A Płucienniczak, H Wedrychowicz. 2007. Enteral vaccination of rats against Fasciola hepatica using recombinant cysteine proteinase (cathepsin L1). Vaccine 25, 36193628.

Mas-Coma S, MD Bargues, MA Valero. 2005. Fascioliasis and other plant-borne trematode zoonoses. Int J Parasitol 35, 1255-1278.

McManus DP, JP Dalton. 2006. Vaccines against the zoonotic trematodes Schistosoma japonicum, Fasciola hepatica and Fasciola gigantica. Parasitology 133, S43-S61.

McVeigh P, AG Maule, JP Dalton, MW Robinson. 2012. Fasciola hepatica virulence-associated cysteine peptidases: a systems biology perspective. Microbes Infect 14, 301-310.

Morphew RM, HA Wright, EJ Lacourse, J Porter, J Barrett, DJ Woods, PM Brophy. 2011. Towards delineating functions within the fasciola secreted cathepsin L protease family by integrating in vivo based sub-proteomics and phylogenetics. PLoS Negl Trop Dis 5, e937.

Mulcahy G, F O'Connor, S McGonigle, A Dowd, DG Clery, SJ Andrews, JP Dalton. 1998. Correlation of specific antibody titre and avidity with protection in cattle immunized against Fasciola hepatica. Vaccine 16, 932-939.

Mulcahy G, F O'Connor, D Clery, SF Hogan, AJ Dowd, SJ Andrews, JP Dalton. 1999. Immune responses of cattle to experimental anti-Fasciola hepatica vaccines. Res Vet Sci 67, 27-33.

Narum DL, SA Ogun, AH Batchelor, AA Holder. 2006. Pas- 
sive immunization with a multicomponent vaccine against conserved domains of apical membrane antigen 1 and 235-kilodalton rhoptry proteins protects mice against Plasmodium yoelii blood-stage challenge infection. Infect Immun 74, 5529-5536.

O’Neill SM, M Parkinson, W Strauss, R Angles, JP Dalton. 1998. Immunodiagnosis of Fasciola hepatica infection (fascioliasis) in a human population in the Bolivian Altiplano using purified cathepsin L cysteine proteinase. Am J Trop Med Hyg 58, 417-423.

Ortiz P, S Scarcella, C Cerna, C Rosales, M Cabrera, M Guzmán, P Lamenza, H Solana. 2013. Resistance of Fasciola hepatica against Triclabendazole in cattle in Cajamarca (Peru): A clinical trial and an in vivo efficacy test in sheep. Vet Parasitol doi: 10.1016/j.vetpar.2013.01.001.

Pérez-Ecija RA, RE Mendes, R Zafra, L Buffonni, A MartínezMoreno, J Pérez. 2010. Pathological and parasitological protection in goats immunised with recombinant cathepsin L1 and challenged with Fasciola hepatica. Vet $J 18$, 351-353.

Piacenza L, D Acosta, I Basmadjian, JP Dalton, C Carmona. 1999. Vaccination with cathepsin L proteinases and with leucine aminopeptidase induces high levels of protection against fascioliasis in sheep. Infect Immun 67, 1954-1961.

Prudencio CR, AO Marra, R Cardoso, LR Goulart. 2010. Recombinant peptides as new immunogens for the control of the bovine tick, Rhipicephalus (Boophilus) microplus. Vet Parasitol 172, 122-131.

Raadsma HW, NM Kingsford, Suharyanta, TW Spithill, D Piedrafita. 2007. Host responses during experimental infection with Fasciola gigantica or Fasciola hepatica in Merino sheep. I. Comparative immunological and plasma biochemical changes during early infection. Vet Parasitol 143, 275-286.

Robinson MW, R Menon, SM Donnelly, JP Dalton, S Ranganathan. 2009. An integrated transcriptomics and proteomics analysis of the secretome of the helminth pathogen Fasciola hepatica: proteins associated with invasion and infection of the mammalian host. Mol Cell Proteomics 8, 1891-1907.

Rokni MB, J Massoud, SM O’Neill, M Parkinson, JP Dalton. 2002. Diagnosis of human fasciolosis in the Gilan province of Northern Iran: application of cathepsin L-ELISA.
Diagn Microbiol Infect Dis 44, 175-179.

Sexton JL, AR Milner, M Panaccio, J Waddington, G Wijffels, D Chandler, C Thompson, L Wilson, TW Spithill, GF Mitchell, NJ Campbell. 1990. Glutathione S-transferase. Novel vaccine against Fasciola hepatica infection in sheep. J Immunol 145, 3905-3910.

Sidhu SS, S Koide. 2007. Phage display for engineering and analyzing protein interaction interfaces. Curr Opin Struct Biol 17, 481-487.

Smith AM, C Carmona, AJ Dowd, S McGonigle, D Acosta, JP Dalton. 1994. Neutralization of the activity of a Fasciola hepatica cathepsin $\mathrm{L}$ proteinase by anti-cathepsin $\mathrm{L}$ antibodies. Parasite Immunol 16, 325-328.

Smooker PM, R Jayaraj, RN Pike, TW Spithill. 2010. Cathepsin B proteases of flukes: the key to facilitating parasite control? Trends Parasitol 26, 506-514.

Spithill, TW, PM Smooker, DB Copeman. 1999. Fasciola gigantica: Epidemiology, control, immunology and molecular biology. In: Dalton JP (ed). Fasciolosis. CABI Publishing, Oxon, UK, Pp 465-525.

Stack C, JP Dalton, MW Robinson. 2011. The phylogeny, structure and function of trematode cysteine proteases, with particular emphasis on the Fasciola hepatica cathepsin L family. Adv Exp Med Biol 712, 116-135.

Villa-Mancera A, H Quiroz-Romero, D Correa, F Ibarra, M Reyes-Pérez, H Reyes-Vivas, G López-Velázquez, K Gazarian, T Gazarian, RA Alonso. 2008. Induction of immunity in sheep to Fasciola hepatica with mimotopes of cathepsin L selected from a phage display library. Parasitology 135, 1437-1445.

Villa-Mancera A, M Méndez-Mendoza. 2012. Protection and antibody isotype responses against Fasciola hepatica with specific antibody to $\mathrm{pIII}$-displayed peptide mimotopes of cathepsin L1 in sheep. Vet J 194, 108-112.

Wijffels GL, L Salvatore, M Dosen, J Waddington, L Wilson, C Thompson, N Campbell, J Sexton, J Wicker, F Bowen, T Friedel, TW Spithill. 1994. Vaccination of sheep with purified cysteine proteinases of Fasciola hepatica decreases worm fecundity. Exp Parasitol 78, 132-148.

Wu HW, XM Hu, Y Wang, JD Kurtis, FJ Zeng, ST McGarvey, GL Wu, ZS Zhang, ZC Hua. 2006. Protective immunity induced by phage displayed mitochondrial related peptides of Schistosoma japonicum. Acta Trop 99, 200-2007. 
